\title{
Necessidades de cuidado de mulheres no climatério com hipertensão: possibilidades de trabalho do enfermeiro*
}

Care needs of women in climacteric with hypertension: nurses' work possibilities

Necesidades de cuidado de mujeres en el climaterio con hipertensión: posibilidades de trabajo del enfermero

\section{Lara Castilhos ${ }^{\mathrm{I}}$, Maria Denise Schimith ${ }^{\mathrm{II}}$, Laís Mara Caetano da Silva ${ }^{\mathrm{III}}$, Lisie Alende Prates ${ }^{\mathrm{IV}}$, Nara Marilene Oliveira Girardon-Perliniv}

Resumo: Objetivo: compreender as necessidades de cuidado de mulheres no climatério com Hipertensão Arterial Sistêmica (HAS). Método: pesquisa qualitativa desenvolvida em 21 Unidades de Saúde da Família (USFs), durante abril e junho de 2019. Participaram 15 mulheres com idade entre 40 e 69 anos; com diagnóstico referido de HAS, adstritas às USFs e que referiram não usar anticoncepcionais hormonais. Utilizou-se entrevista semiestruturada, seguida pela construção e devolução de narrativas, com análise participativa. Resultados: as necessidades de cuidado incluíam manejo dos sinais e sintomas; acompanhamento dos níveis pressóricos, efetividade e adesão ao tratamento; orientação sobre escolhas alimentares; busca por informações confiáveis e escuta ativa. A percepção acerca do trabalho do enfermeiro remeteu ao atendimento individual e pontual. Conclusão: o climatério é vivenciado de forma distinta e as necessidades das mulheres demonstram diferentes possibilidades de aprimoramento do cuidado. Essa identificação permite que o enfermeiro desenvolva atenção individualizada e adaptada às demandas das mulheres.

Descritores: Climatério; Hipertensão; Saúde da Mulher; Enfermagem; Atenção Primária à Saúde

Abstract: Objective: to understand the care needs of women in climacteric with Systemic Arterial Hypertension (SAH). Method: this is qualitative research developed in 21 Family Health Units (FHUs), during April and June

\footnotetext{
Enfermeira. Mestre em Enfermagem. Instituto Federal Catarinense. Santa Rosa do Sul, Santa Catarina, Brasil. E-mail: laracastilhos23@gmail.com - ORCID: https://orcid.org/0000-0003-2845-973X.

II Enfermeira. Pós-doutora em Enfermagem/FURG. Professora Adjunta da Universidade Federal de Santa Maria. Santa Maria, Rio Grande do Sul, Brasil. E-mail: ma.denise2011@gmail.com - ORCID: http://orcid.org/0000-0002-4867-4990

III Enfermeira, Pós-doutora em Enfermagem em Saúde Pública. Professora Adjunta da Universidade Federal de Santa Maria. Santa Maria, Rio Grande do Sul, Brasil. ORCID: https://orcid.org/0000-0001-7596-2333.

IV Enfermeira, Doutora em Enfermagem, Professora Adjunta da Universidade Federal do Pampa. Uruguaiana, Rio Grande do Sul, Brasil. ORCID: http://orcid.org/0000-0002-5151-0292.

V Enfermeira, Pós-doutora em Enfermagem, Professora Associada, Universidade Federal de Santa Maria, Santa Maria, Rio Grande do Sul, Brasil, E-mail: nara.girardon@gmail.com ORCID: 0000-0002-3604-2507

* Extraído da dissertação "Necessidades de cuidado de mulheres em climatério com hipertensão: possibilidades de atuação do enfermeiro", Programa de Pós-Graduação em Enfermagem, Universidade Federal de Santa Maria, 2020.
} 
Necessidades de cuidado de mulheres no climatério com hipertensão: possibilidades de... $\mid 2$

2019. Fifteen women between 40 and 69 years old participated; with a referred diagnosis of SAH, attached to the FHUs and who reported not using hormonal contraceptives. We used semi-structured interviews, followed by the construction and return of narratives, with participatory analysis. Results: care needs included management of signs and symptoms; monitoring blood pressure levels, effectiveness, and treatment adherence; guidance on food choices; search for reliable information, and active listening. The perception of the nurse's work referred to individual and punctual care. Conclusion: the climacteric is experienced differently and the needs of women show different possibilities for improving care. This identification allows the nurse to develop individualized care and adapted to the demands of women.

Descriptors: Climacteric; Hypertension; Women's Health; Nursing; Primary Health Care

Resumen: Objetivo: comprender las necesidades de cuidado de mujeres en el climaterio con Hipertensión Arterial Sistemica (HAS). Método: investigación cualitativa desarrollada en 21 Unidades de Salud de la Familia (USFs), durante abril y junio de 2019. Participaron 15 mujeres con edad entre 40 y 69 años; con diagnóstico referido de HAS, adscritas a las USFs y que dijeron no usar anticoncepcionales hormonales. Se utilizó una entrevista semiestructurada, seguida por la construcción y devolución de narrativas, con análisis participativa. Resultados: las necesidades de cuidado incluyeron el manejo de señales y síntomas; acompañamiento de los niveles de presión, efectividad y adhesión al tratamiento; orientación sobre elecciones alimentares; búsqueda por informaciones confiables y escucha activa. La percepción acerca del trabajo del enfermero fue sobre el atendimiento individual y puntual. Conclusión: el climaterio es vivido de forma distinta y las necesidades de las mujeres demuestran diferentes posibilidades de mejora del cuidado. Esa identificación permite que el enfermero desarrolle una atención individualizada y adaptada a las demandas de las mujeres.

Descriptores: Climaterio; Hipertensión; Salud de la Mujer; Enfermería; Atención Primaria de Salud

\section{Introdução}

As mulheres compõem a maior parcela da população brasileira, representando $51,7 \%$ do total de habitantes do país. ${ }^{1}$ Elas também são as principais usuárias do Sistema Único de Saúde (SUS), e tal representatividade tem exigido novas perspectivas na atenção voltada à sua saúde, com o objetivo de estruturar uma assistência que as englobe na sua totalidade. A partir disso, foi elaborada a Política Nacional de Atenção Integral à Saúde da Mulher (PNAISM), ${ }^{2}$ a qual procurou preencher as lacunas deixadas por políticas anteriores em relação a determinados períodos/grupos do ciclo vital feminino, por exemplo, as mulheres no climatério. Por climatério, entende-se uma condição endócrina, caracterizada pelo declínio progressivo de estrogênio, resultante da depleção dos folículos ovarianos. Essa fase compreende a transição entre o período 
reprodutivo e não reprodutivo, que se manifesta entre os 35 e 40 anos, podendo se estender aos 65 anos de idade. ${ }^{3}$

A PNAISM representou um avanço para a saúde feminina ao incorporar a integralidade do cuidado e a promoção da saúde como princípios norteadores, especialmente para as mulheres que vivenciam o climatério, servindo, por exemplo, como subsídio para a elaboração do Manual Atenção Integral à Saúde da Mulher no Climatério/Menopausa. Contudo, a atenção à saúde nesta etapa da vida ainda não abrange a integralidade desejada, tendo em vista que as demandas desse grupo nem sempre são materializadas na Atenção Primária à Saúde (APS), ponto da Rede de Atenção à Saúde (RAS) que seria o mais apropriado para essa assistência. ${ }^{4}$

Portanto, embora sejam notáveis os avanços na área, dada a incipiência desse movimento no âmbito das políticas e ações programáticas do Ministério Saúde, as ações das políticas e programas ainda se encontram centralizadas na área materno-infantil. Ademais, ainda persiste a ideia, na sociedade em geral, de que, ao chegar no climatério, a mulher tem a finitude de fecundidade e de sua capacidade produtiva, o que representaria o fim da sexualidade, além de outras questões. ${ }^{2}$ Dessa forma, permanecem ações de saúde fragmentadas e reduzidas para as mulheres no climatério, e mais ampliadas e contextualizadas para as mulheres em período reprodutivo.

Dentro dessa lógica, a centralização de ações no período gravídico-puerperal tem implicado na reserva dos recursos em saúde para essa área, mantendo em segundo plano as estratégias direcionadas para as mulheres no climatério. Desse modo, o Manual Atenção Integral à Saúde da Mulher no Climatério/Menopausa alerta para a necessidade de esse grupo ser incluído no planejamento estratégico, técnico e financeiro, com foco no investimento em ações de saúde. ${ }^{4}$ As mulheres nesse período correspondem a 15,5\% da população feminina brasileira, o que representa, aproximadamente, um contingente de 16,21 milhões de pessoas, ${ }^{5}$ justificando a necessidade de ações que considerem as suas especificidades e necessidades. 
Necessidades de cuidado de mulheres no climatério com hipertensão: possibilidades de... | 4

Também é válido destacar algumas doenças crônico-degenerativas que surgem em função das alterações decorrentes do climatério, como a Hipertensão Arterial Sistêmica (HAS), que incide a partir da diminuição do hormônio estrogênio, o qual é responsável pela proteção dos vasos sanguíneos da mulher. ${ }^{6}$ Portanto, considerando que o hipoestrogenismo gradual é uma característica inerente ao climatério, a HAS constitui uma doença crônica não transmissível (DCNT), que tem demonstrado níveis mais elevados em mulheres a partir da quinta década de vida. ${ }^{7}$ Ademais, a HAS consiste em problema de saúde pública que está diretamente implicado no surgimento de doenças cardiovasculares, as quais constituem as principais causas de mortalidade em todo o mundo. ${ }^{8}$

Logo, se tratando de mulheres, as alterações hormonais, circulatórias e sanguíneas decorrentes do climatério podem potencializar os riscos cardiovasculares, o que reforça a necessidade de uma atenção integral a esse grupo, avaliando precocemente fatores de risco cardiovascular, com o intuito de reduzir a morbidade e a mortalidade ${ }^{6}$ Assim, emerge o cuidado às mulheres no climatério como uma demanda no campo da promoção da saúde e do cuidado longitudinal na APS. ${ }^{9}$ Nessa perspectiva, é fundamental o trabalho do enfermeiro, uma vez que ele é responsável por articular, coordenar e conduzir as práticas de cuidado. ${ }^{10}$

Contudo, essas práticas precisam ir além da execução de conhecimentos técnicos, sendo necessário que contemplem o acolhimento, o vínculo, a responsabilização e a resolutividade. É imprescindível a articulação entre diferentes práticas que compõem o arcabouço organizativo do SUS, independentemente da densidade tecnológica empregada. ${ }^{11}$

Desse modo, o presente estudo buscou responder a seguinte questão de pesquisa: como as mulheres no climatério com HAS adstritas à Estratégia de Saúde da Família identificam suas necessidades de cuidado? Tem-se como objetivo: compreender as necessidades de cuidado de mulheres no climatério com HAS. 
5 | Castilhos L, Schimith MD, Silva LMC, Prates LA, Girardon-Perlini NMO

\section{Método}

Trata-se de um estudo qualitativo. A pesquisa qualitativa possibilita a interpretação dos fenômenos, a descrição e a análise da realidade, ${ }^{12}$ assim como as experiências, sintomas e necessidades vivenciadas pelas mulheres em climatério com HAS. Consistiram em cenário do estudo todas as Unidades de Saúde da Família (USFs) de um município localizado no Sul do Brasil, englobando, assim, 21 serviços.

A seleção das participantes deu-se de forma intencional. Elas foram identificadas a partir do banco de dados vinculado ao projeto matricial que envolvia a estratificação do risco cardiovascular dos usuários atendidos na Atenção Básica do município, com autorização da pesquisadora responsável. Utilizaram-se como critérios de seleção: mulheres com idade entre 40 a 69 anos, devido ao banco do projeto matricial estar dividido por faixas etárias de dez em dez anos; com diagnóstico referido de HAS, adstritas às USFs e que referiram não usar anticoncepcionais hormonais, totalizando 42 mulheres.

Destas, 15 mulheres foram entrevistadas, visto que 16 não aceitaram participar do estudo, e nove não foram localizadas via telefone e endereço. Ressalta-se que foram realizadas três tentativas telefônicas e dois deslocamentos até o seu domicílio. Optou-se por não ter o acompanhamento do Agente Comunitário de Saúde, com o intuito de propiciar privacidade às mulheres. Em um caso o marido respondeu pela mulher, não permitindo sua participação, e uma foi a óbito. A coleta de dados ocorreu no período do compreendido entre abril e junho de 2019. O contato inicial com as participantes ocorreu por meio telefônico, convidando-as a agendar a entrevista e definir o local em que ela ocorreria. As mulheres optaram por realizá-las em seus domicílios (14) ou na USF (uma), o que foi acatado pela pesquisadora.

Durante a entrevista, realizada por um único entrevistador, utilizou-se o termo “menopausa” ao invés de “climatério”, e o termo “pressão alta” no lugar de "HAS”, com o intuito de facilitar o entendimento das participantes. Destaca-se que durante a entrevista esses 
Necessidades de cuidado de mulheres no climatério com hipertensão: possibilidades de... $\mid 6$

conceitos foram esclarecidos. As entrevistas foram compostas por duas etapas: a primeira com dados referentes à caracterização sociodemográfica das participantes e a segunda com questões que versaram sobre a vida após o início dos sintomas, os cuidados no período do climatério, hipertensão e quem auxiliava nesse processo; as dúvidas e quem as ajudava as compreender melhor; as necessidades de cuidado; como o enfermeiro poderia ajudar; as expectativas em relação à USF e ao enfermeiro; a opinião acerca de um grupo de saúde que abordasse esses temas, bem como dia e horário possíveis para retornar a entrevista. A duração média de cada entrevista foi de 30 minutos, e estas foram registradas em um gravador digital do tipo MP3 Player ${ }^{\circ}$.

Utilizou-se a análise participativa, a qual segue as etapas de transcrição das entrevistas; confecção das narrativas e retorno das narrativas para as participantes. ${ }^{13}$ Após a transcrição, construíram-se narrativas individuais, conduzidas como uma história, que foram apresentadas às participantes, conforme horário e local de suas preferências. No retorno das narrativas, a história foi lida em voz alta, oportunizando correções ou supressões. A devolução foi registrada em gravador digital do tipo MP3 Player $^{\circ}$, e teve duração média de 45 minutos. Salienta-se que, entre a entrevista e a devolução da narrativa, houve um intervalo de, no mínimo, duas semanas, a fim de obter maior autenticidade nas respostas. ${ }^{13}$ Esse processo foi aplicado com todas as participantes. A organização dos núcleos argumentais, utilizando as transcrições das narrativas, contou com o auxílio do software QSR NVivo $12 \mathrm{PRO}^{\circ}$, disponível na versão gratuita.

O estudo respeitou os preceitos éticos das Resoluções no 466/2012 e no 510/2016, do Conselho Nacional de Saúde. O projeto foi aprovado pelo Comitê de Ética em Pesquisa em 11 de janeiro de 2019, sob o Parecer n. 3.111.239 e CAAE no 05333118.2.0000.5346. O Termo de Consentimento Livre e Esclarecido foi apreciado e assinado por todas as participantes. Para manter o seu anonimato, os nomes das mulheres foram substituídos pela letra "N", a qual identifica o processo da narrativa, e os números de 01 a 15, formando códigos, com exemplo: N-01. 


\section{Resultados}

Participaram do estudo 15 mulheres em climatério e com HAS, residentes em áreas adstritas às USFs. Destas, oito (53,3\%) possuíam entre 50 e 59 anos de idade, e houve quatro $(26,6 \%)$ de 40 a 49 anos e três $(20,0 \%)$ de 60 a 65 anos.

Quanto à escolaridade, oito $(53,3 \%)$ estudaram entre zero a oito anos, seis $(40,0 \%)$, entre nove a 11 anos, e uma (6,6\%) estudou por 12 anos ou mais. Ao tratar da ocupação, nove $(60,0 \%)$ eram assalariadas ou autônomas, quatro (26,6\%) eram donas de casa, uma (6,6\%) estava desempregada e uma era pensionista $(6,6 \%)$. Sete $(46,6 \%)$ eram legalmente casadas, cinco $(33,3 \%)$ eram divorciadas, uma $(6,6 \%)$ era solteira, uma $(6,6 \%)$ estava em união estável, e outra participante $(6,6 \%)$ era viúva.

Ao tratar da etnia, sete $(46,6 \%)$ se autodeclararam brancas, cinco $(33,3 \%)$ eram pardas, ao passo que três $(20,0 \%)$ eram negras. Quanto à renda, sete $(46,6 \%)$ recebiam entre um e dois salários mínimos, quatro $(26,6 \%)$ recebiam de dois a três salários mínimos, três $(20,0 \%)$ recebiam até um salário mínimo e uma (6,6\%) recebia mais de cinco salários mínimos. Ao serem questionadas sobre a religião, $12(80,0 \%)$ responderam serem católicas e três $(20,0 \%)$, evangélicas.

A compreensão acerca das necessidades de cuidado das mulheres no climatério com HAS perpassa pela identificação dos sinais e sintomas relacionados ao climatério. Tais manifestações foram percebidas com intensidades distintas pelas mulheres, a partir de suas percepções e vivências individuais, visto que algumas perceberam os sinais e sintomas de forma mais intensa, ao passo que as alterações inerentes a esse período não foram percebidas por outras.

Eu tenho esse calorão, essas coisas horríveis. É horrível essa menopausa. Eu não sei também, mas uma vez fui no ginecologista, e ele disse que quem tem diabetes não pode fazer tratamento para menopausa, aí eu não tomo nada [...]. E aí minha vida mudou bastante. (N-01)

Quando a menopausa começou eu tive que tirar o útero, os ovários e as trompas, porque eu tive miomas por dentro e por fora. Mas continua aqueles calorões, suor e a pressão alta. $(\mathrm{N}-02)$ 
Necessidades de cuidado de mulheres no climatério com hipertensão: possibilidades de... $\mid 8$

Eu sinto muito calorão, muita ansiedade. Tem noites que eu demoro para pegar no sono. Eu sinto que eu estou irritada. Dá aquela dor de cabeça, e eu desligo tudo em casa. Faço as minhas coisas e vou deitar na cama e fico bem quieta. Brigo até com os bichos. (N-03)

Eu nunca tive os calorões. Só depois dos 40 que começa a dor aqui, dor ali, mas eu acho que não é da menopausa, mas não de ter aqueles calorões. (N05)

A partir dos relatos apresentados, é possível identificar que o "passar pela menopausa” repercute em experiências distintas para as mulheres, haja vista que os sinais e sintomas podem apresentar intensidades variadas. Dessa forma, as necessidades de cuidado decorrentes do climatério demandam um planejamento singular do cuidado prestado, com o intuito de que este seja resolutivo, contínuo e acessível para a mulher.

Além dos sinais e sintomas apresentados nos relatos anteriores, as mulheres conviviam com mudanças corporais. Dentre estas, verificou-se, por exemplo, o ganho de peso.

Meu peso mudou bastante[...] de 72 para 95. (N-02)

Eu pesava 64-65, e agora eu peso 70, e não tem jeito mais, eu caminho e danço, e não tem jeito [...]. (N-09)

Vejo mudanças no corpo, mudou tudo, o organismo não é o mesmo. Meu peso aumentou cerca de 10 quilos. (N-15)

Alterações no humor também são sintomas comuns do climatério. Entretanto, ao mesmo tempo que algumas participantes relataram alterações de humor significativas, outras não as perceberam. Isso remete ao fato de que, em razão da singularidade do período, as mulheres se percebem de formas diversas, conforme apontam os relatos.

Meu humor mudou, agora estou sempre estressada [...]. (N-10) 
9 | Castilhos L, Schimith MD, Silva LMC, Prates LA, Girardon-Perlini NMO

Meu humor mudou, dá uma depressão [...]. Uma hora eu estou bem, e daqui a pouco muda. Eu fiquei mais esquecida, e a psicóloga até falou que era do estresse. $(\mathrm{N}-03)$

Em relação ao meu humor não mudou muita coisa. $(\mathrm{N}-11)$

Meu humor continuou o mesmo [...] não alterou. (N-04)

Em relação ao trabalho, as participantes relataram cansaço e mudança no ritmo de realização das tarefas laborais. Conforme se constatou, essas alterações resultaram na saída do mercado de trabalho.

Eu noto que no trabalho mudei o meu ritmo. Eu sou muito mais devagar para fazer as coisas. E às vezes eu me atrapalho, perco, esqueço muito as coisas. $(\mathrm{N}-03)$

Eu larguei o trabalho [...] porque eu cansava muito fácil[...]. ( $\mathrm{N}-10)$

Compreender como as mulheres no climatério convivem com a HAS oferta indícios importantes acerca das suas necessidades de cuidado. Aspectos relevantes, e que repercutem no planejamento do cuidado, incluíram o desconforto relacionado ao tratamento contínuo e as dificuldades de adesão à terapêutica medicamentosa prescrita em longo prazo.

O que modificou na minha vida é que eu tenho que tomar o remédio sempre, que eu tenho que me cuidar. $(\mathrm{N}-11)$

Eu estou tomando remédio. Eu tomo losartana, e me sinto bem, porque a pressão normaliza. $(\mathrm{N}-04)$

Tem dias que ela está em um pico alto, e eu tomo remédio diariamente, e tem horas que eu me canso dos remédios, de tanto que eu tomo. E aí eu paro [o medicamento] e ela se vai e volta [...]. ( $\mathrm{N}-08)$

Eu acho tranquilo [...] eu não faço questão de tomar remédio todos os dias, aí um dia fui na unidade [USF] e disseram que era pressão, aí eu tomo medicação, hidroclorotiazida $25 \mathrm{~g}$, que é bem fraquinho [...]. (N-12) 
Necessidades de cuidado de mulheres no climatério com hipertensão: possibilidades de... $\mid 10$

Ao tratar da alimentação, as mulheres relataram que, ao mesmo tempo em que não conseguiam alimentar-se adequadamente, buscavam ajustar o horário das refeições. Foram relatadas práticas como a ingestão de legumes e verduras e a diminuição da ingestão de alimentos ricos em lipídios, entretanto, o controle em relação ao uso do sódio nas refeições permanecia como um desafio.

Eu preciso ter mais cuidado na alimentação [...] baixar o sal. (N-08)

Eu tenho muita azia, então na alimentação eu evito comer gordura [...]. (N09)

Eu me alimento muito mal, eu não consigo comer ao meio-dia, aí como qualquer lanchinho e saio, de manhã não consigo tomar café, só de noite que eu me alimento melhor. $(\mathrm{N}-12)$

Eu como arroz, um pouco de feijão, salada, verdura, porque a gente entra na alimentação da minha mãe e a gente continua [...]. ( $\mathrm{N}-13)$

Quanto à conduta adotada ao apresentar-se alguma dúvida ou insegurança em relação ao climatério ou à HAS, as mulheres relataram buscar informações em fontes diversas, como médicos, familiares, amigos e Internet. Apesar de relatos acerca desse processo de busca, algumas participantes disseram preferir não saber o que ocorria com o seu corpo.

Eu sempre pergunto muito as coisas ao médico, gosto de estar bem informada. $(\mathrm{N}-02)$

Eu esclareço na Internet. Eu leio muito sobre as doenças, tudo [...] (N-08)

Se vai escutar uma coisa que vai me deixar confusa e não está com boa vontade para explicar, então eu prefiro ficar com aquilo ali sem eu saber, do que a pessoa me botar dúvida e eu ainda me magoar. $(\mathrm{N}-12)$

Em razão da contribuição que o enfermeiro pode oferecer no período do climatério e no manejo da HAS, questionou-se às mulheres acerca do trabalho desse profissional na USF a que a 
11 | Castilhos L, Schimith MD, Silva LMC, Prates LA, Girardon-Perlini NMO

participante era vinculada. Os relatos expressaram a aproximação a esse profissional em circunstâncias como a possibilidade de acesso à consulta médica, a realização de exame citopatológico, a oferta de orientações quando da realização do exame citopatológico e a aferição dos níveis pressóricos.

Eu já consultei com a enfermeira [...] a gente passa primeiro com elas para depois chegar com o médico. $(\mathrm{N}-10)$

Eu não procuro a enfermeira para conversar. Mas eu sempre faço meu preventivo com a enfermeira e ela sempre me dá uma orientação. (N-11)

Eu nunca conversei com a enfermeira sobre isso, porque eu só procurei a doutora, e então ela disse que era menopausa, mas nunca tive acompanhamento da enfermeira. $(\mathrm{N}-13)$

O que falta ali são mais médicos. Tem médico, mas é difícil conseguir ficha. $E$ agora está faltando ginecologista, não sei por que não tem. Com a enfermeira eu nunca conversei [...] o enfermeiro tem que esclarecer mais as coisas para a gente. $(\mathrm{N}-14)$

A enfermeira ajuda [...] ela verifica a pressão da gente antes e depois do exercício. Ela tem o papelzinho aquele, então vai direto. A enfermeira me explicou o que era menopausa. (N-09)

Identificou-se, a partir dos resultados apresentados, que as mulheres em climatério e com HAS apresentavam necessidades de cuidado. Dentre as principais demandas sinalizadas, estão aspectos como: o manejo dos sinais e sintomas decorrentes do climatério (fogachos, sudorese, ansiedade, alterações de humor, aumento de peso e fadiga); o acompanhamento dos níveis pressóricos, da efetividade da terapêutica medicamentosa e da adesão ao tratamento prescrito; a orientação acerca de escolhas alimentares acessíveis e saudáveis; a importância da busca por informações provenientes de fontes confiáveis; a realização de escuta ativa, que repercuta em orientações compreensíveis pela mulher, pautada em uma relação horizontalizada. 
Necessidades de cuidado de mulheres no climatério com hipertensão: possibilidades de... $\mid 12$

\section{Discussão}

As participantes deste estudo possuíam, em sua maioria, idade entre 50 e 59 anos. Quanto à escolaridade, a maioria estudou entre zero e oito anos, eram assalariadas ou autônomas, legalmente casadas, brancas e recebiam de um a dois salários mínimos, o que coaduna com estudo realizado na cidade de Prudente, no Estado de São Paulo, o qual identificou que as mulheres no climatério apresentavam, em média, 51 anos, tinham ensino fundamental incompleto, eram casadas, brancas e possuíam renda de até três salários mínimos. ${ }^{14}$

As entrevistadas referiram sinais e sintomas como fogachos, aumento da sudorese, cefaleia, ansiedade, irritabilidade e aumento da PA, relacionando-os ao climatério. Ele é um período complexo na vida da mulher, em especial devido às mudanças biopsicossociais decorrentes desta fase, cujos sintomas, se não controlados, impactam negativamente nas atividades cotidianas das mulheres. ${ }^{15}$ Tais sintomas podem refletir em manifestações genitais (redução de libido), extragenitais (atrofia e distrofia da vulva, dor, secura e sangramento vaginal) e psíquicas (fogachos, suor, cefaleia, cansaço, fraqueza, irritabilidade, alteração do humor e depressão), o que se assemelha aos relatos das participantes deste estudo. ${ }^{16}$

As participantes relataram aumento de peso e dificuldades em realizar uma alimentação saudável, o que inclui fazê-lo em horários planejados e fazer uma ingesta nutritiva e calórica adequada. O hábito alimentar inadequado repercute em mudanças corporais que, por sua vez, impactam sobremaneira na alteração da imagem corporal, refletindo negativamente na percepção de seu corpo, o que pode interferir no estado da saúde psíquica. ${ }^{17}$

As mudanças no peso e alterações de humor, assim como o cansaço físico no trabalho, mencionados pelas participantes, podem estar relacionados às mudanças nas condições de vida das mulheres. Tais alterações foram significativas nas últimas décadas, como o aumento da entrada das mulheres no mercado de trabalho e a inversão de papéis sociais, ${ }^{18}$ que pode resultar em sobrecarga para elas, gerando cansaço físico e esgotamento mental. Considerando que essas mulheres estão 
com idade entre 50 e 65 anos, a fadiga também pode estar relacionada com o aumento da idade, concomitante às alterações fisiológicas e hormonais. Nesse sentido, percebe-se que este sintoma pode ser agravado conforme as atividades laborais, alimentação e regulação do sono.

As participantes referiram que o estresse, a ansiedade, o desânimo, a perda de memória, a falta de concentração e alterações repentinas de humor são eventos e sensações inerentes a essa fase. A mudança de humor é um sintoma que pode ocorrer durante o processo fisiológico do climatério, pois nesse período ocorre a diminuição do hormônio estrogênio, o que suscita significativamente a prevalência de depressão, reatividade emocional e mudanças cognitivas que podem ser relacionadas a maior prevalência de irritabilidade, estresse, tristeza e depressão. ${ }^{19}$

Identificou-se, ainda, que as mulheres em climatério com HAS apresentavam dificuldades quanto à adesão à terapia medicamentosa da HAS a longo prazo, e não compreendiam sua importância. As dificuldades relacionadas à adesão ao tratamento podem ser decorrentes de possíveis receios em relação ao uso da medicação e à cronicidade da doença, o que pode resultar em elevação do risco para o desenvolvimento de eventos cardiovasculares, como infarto agudo do miocárdio e acidente vascular encefálico, por exemplo. Dessa forma, a adesão ao tratamento se constitui como mais uma necessidade de cuidado a ser contemplada pelo enfermeiro.

Acerca disso, a literatura afirma que a baixa escolaridade, a idade e a irregularidade no comparecimento às consultas ginecológicas contribuem para a não compreensão do climatério, de seus sinais e sintomas e seu manejo/tratamento. ${ }^{20}$ Estudo que analisou o perfil de mulheres com HAS traz que a menor renda salarial pode ser apontada como um problema no processo de adesão ao tratamento e, consequentemente, no controle dos níveis pressóricos, ${ }^{8}$ corroborando com os achados do presente estudo.

A dificuldade de aderir ao tratamento medicamentoso resulta em descompensação da pressão arterial, o que resulta em elevação do risco de eventos cardiovasculares. ${ }^{8}$ Nesse sentido, 
Necessidades de cuidado de mulheres no climatério com hipertensão: possibilidades de... | 14

os cuidados a serem ofertados para essa parcela da população devem abranger a educação em saúde, prática na qual torna-se necessário o enfermeiro identificar as potencialidades e as dificuldades da mulher em compreender seu diagnóstico. Consequentemente, cuidar de sua condição clínica, realizando orientações acerca das medicações a serem utilizadas, seus benefícios e efeitos colaterais, além dos possíveis impactos da falta de adesão sobre a patologia apresentada.

A partir da análise dos relatos das mulheres em climatério com HAS, identificaram-se como necessidades de cuidado o manejo dos sinais e sintomas decorrentes do climatério, entre eles o ganho de peso, mudança no humor, alteração no ritmo de trabalho devido ao cansaço físico, bem como o acompanhamento do nível pressórico, da efetividade da terapêutica medicamentosa e da adesão ao tratamento prescrito. Também foram identificadas a necessidade de orientação acerca de escolhas alimentares acessíveis e saudáveis, a importância da busca por informações provenientes de fontes confiáveis, a realização de escuta ativa e de orientações compreensíveis e acessíveis para esse recorte populacional.

Ao tratar da atenção à saúde da mulher no climatério com HAS, é preciso refletir acerca do trabalho a ser desenvolvido pelo enfermeiro, sendo necessário aprimorar as práticas e desenvolver um cuidado alinhado ao contexto específico vivenciado por essas mulheres. O cuidado precisa pautar-se nos aspectos sociais, históricos, econômicos e culturais, bem como nas necessidades e especificidades da mulher, haja vista a experiência singular em relação a essa fase da vida. ${ }^{21}$

Portanto, o enfermeiro deve desenvolver ações de promoção e educação em saúde, bem como ações voltadas à prevenção de doenças, proporcionando às usuárias da USF espaços coletivos para troca de conhecimento, com o intuito de sanar dúvidas referentes à alimentação saudável e acessível tanto às suas necessidades nutricionais, quanto às condições socioeconômicas. ${ }^{16}$ Ainda, podem ser realizadas orientações acerca da atividade física regular, pois tal prática possui efeito positivo sobre as paredes dos vasos sanguíneos e apresenta ação 
vasodilatadora, contribuindo com o envelhecimento saudável e prevenindo a manifestação de comorbidades como HAS no período do climatério. ${ }^{18}$

O presente estudo permitiu identificar que os sintomas comuns e fisiológicos do climatério nem sempre são vivenciados por todas as mulheres. Diversos fatores estão relacionados às percepções acerca da sintomatologia, como as condições socioeconômicas, a etnia, o estado civil e a qualidade das relações familiares. ${ }^{22}$ Logo, o cuidado precisa ser individualizado e adaptado às vivências e condições de cada mulher. Evidencia-se a importância de que o enfermeiro tenha profundo conhecimento acerca das características da população adstrita à USF, para que a oferta de cuidado seja planejada a partir das necessidades de cada uma das mulheres e se consiga promover um cuidado integral e equânime.

Ainda, a percepção acerca do trabalho do enfermeiro remeteu a formas de cuidado desenvolvidas por esse profissional, predominantemente alinhadas à perspectiva individual do cuidado. Nesse sentido, percebeu-se que as mulheres o identificaram a partir da realização do acolhimento para posterior encaminhamento à consulta médica. Ainda mencionaram que esse profissional desenvolvia as consultas voltadas à realização de exame citopatológico e do fornecimento de orientações relacionadas ao exame e sua condição de saúde, além do monitoramento dos níveis pressóricos. Esses indícios demonstram que o trabalho do enfermeiro está relacionado à prática clínica e à realização de ações de educação individuais, como orientações.

A partir das percepções identificadas, torna-se necessário que o enfermeiro amplie seu campo de visão, com o intuito de materializar o cuidado, tanto individual quanto coletivo, incluindo as mulheres que residem na área adstrita à USF. Para isso, deve criar vínculos e compreender as necessidades de cuidado de cada usuária, promovendo ações pautadas na realização de orientações compreensíveis e acessíveis. Evidencia-se que o enfermeiro precisa criar um espaço de atendimento para a mulher no climatério, com o intuito de oportunizar a escuta qualificada dos sentimentos e anseios inerentes a essa fase da sua vida. O profissional 
Necessidades de cuidado de mulheres no climatério com hipertensão: possibilidades de... | 16

pode oferecer orientações, informações sobre as modificações e suporte emocional, de modo a minimizar o desconforto decorrente de alterações desagradáveis e possíveis implicações negativas para a saúde. Pode, ainda, contribuir para desconstruir mitos e preconceitos, incentivando reflexões que desconstruam estereótipos que limitem a vivência plena da mulher no climatério com HAS, inclusive sua sexualidade. ${ }^{23}$

Ainda, ao realizar a consulta de enfermagem com mulheres em idade próxima ao climatério, o enfermeiro precisa trazer informações relacionadas às DCNTs (por exemplo: HAS, Diabetes Mellitus e dor crônica). Com isso, é possível orientar os possíveis sinais e sintomas decorrentes destas patologias, além de discorrer sobre o climatério, buscando fazer com que estas mulheres vivenciem esse processo de forma mais tranquila. ${ }^{24-25}$

Pretende-se, dessa forma, colaborar com a práxis do enfermeiro no cuidado à mulher no climatério com HAS, demonstrando a necessidade de planejamento e implementação de práticas de cuidado integrais. A partir dessa perspectiva, pode-se conhecer a mulher em sua totalidade, compreendendo-a como alguém que se constitui como resultado de suas diferentes vivências, auxiliando o enfermeiro a desenvolver o cuidado a partir de sua práxis profissional.

Se constituem como limitações do estudo a possível interferência ocorrida a partir da participação de um ou mais familiares das mulheres no momento da entrevista. Algumas mulheres optaram por responder a entrevista e realizar a devolução da narrativa no local de trabalho, o que pode ter influenciado no tempo disponível e na qualidade das respostas fornecidas. Ainda, há um limite no que tange à discussão dos resultados, visto que não foram identificados estudos que versassem acerca do climatério e da HAS de maneira integrada. Sugere-se, por fim, o desenvolvimento de novos estudos que busquem aprofundar na compreensão desta fase específica da vida da mulher, ampliando a perspectiva de que o cuidado da mulher deva se restringir ao período fértil e puerperal. 


\section{Conclusão}

O estudo em tela evidencia que a experiência relacionada ao climatério se dá de forma distinta entre as mulheres, e que a presença da HAS nessa fase da vida se constitui como uma realidade desafiadora para as próprias mulheres e para os enfermeiros que trabalham nas USFs. As participantes demonstraram ter pouco conhecimento acerca do climatério e algum conhecimento sobre a HAS e suas repercussões, sinalizando para a necessidade de adaptação dos hábitos alimentares e o manejo do estresse. Entretanto, a adesão à terapêutica medicamentosa permaneceu como uma condição complexa, remontando à necessidade de o enfermeiro ampliar suas práticas para ações de promoção da saúde, prevenção de doenças e educação em saúde.

Ainda tratando do trabalho do enfermeiro, as mulheres identificaram que esses profissionais realizavam o cuidado de forma individual e pontual, sendo necessária uma ampliação da forma como o cuidado era produzido. Esse achado pode ser considerado umas das contribuições deste estudo, tendo em vista que pode servir como subsídio para o enfermeiro na implementação de estratégias que permitam a prevenção de agravos e a promoção da saúde de mulheres no climatério com hipertensão, bem como a redução da morbidade e mortalidade por DCNTs. Dessa forma, sugere-se a incorporação de práticas como visitas domiciliares, realização de grupos, dentre outras, que partam da perspectiva de horizontalização das relações e oportunizem a troca de experiências e conhecimentos entre as mulheres e os profissionais.

\section{Referências}

1. Instituto Brasileiro de Geografia (IBGE). Pesquisa nacional por amostra de domicílios contínua 2018 [Internet]. Brasília (DF): IBGE; 2018 [acesso em 2020 jan 10]. Disponível em: https://educa.ibge.gov.br/jovens/conheca-o-brasil/populacao/18320-quantidade-de-homens-e mulheres.html

2. Ministério da Saúde (BR). Política Nacional de Atenção Integral à Saúde da Mulher: princípios e 
Necessidades de cuidado de mulheres no climatério com hipertensão: possibilidades de... $\mid 18$

diretrizes. Brasília (DF): Ministério da Saúde; 2004.

3. Silva VH, Rocha JSB, Caldeira AP. Fatores associados à autopercepção negativa de saúde em mulheres climatéricas. Ciênc Saúde Colet. 2018;23(5):1611-20. doi: 10.1590/1413-81232018235.17112016.

4. Ministério da Saúde (BR). Manual de atenção à mulher no climatério/menopausa. Brasília (DF): Ministério da Saúde; 2008.

5. Instituto Brasileiro de Geografia e Estatística (IBGE). Contagem da População 2015 [Internet]. Brasília (DF): IBGE; 2015 [acesso em 2020 jan 15]. Disponível em: http://www.ibge.gov.br/home/estatitica/população/domcavati/defaut.shtm

6. Melo JB, Campos RCA, Carvalho PC, Meireles MF, Andrade MVG, Rocha TPO, et al. Fatores de risco cardiovasculares em mulheres climatéricas com doença arterial coronariana. Int J Cardiovasc Sci. 2018;31(1):4-11. doi: 10.5935/2359-4802.20170056

7. Sociedade Brasileira de Cardiologia; Sociedade Brasileira de Hipertensão; Sociedade Brasileira de Nefrologia. VI Diretrizes brasileiras de hipertensão. Arq Bras Cardiol. 2010;95(1 Suppl 1):1-51. doi 10.1590/S0066-782X2010001700001

8. Silva SSBE, Oliveira SFSB, Pierin AMG. The control of hypertension in men and women: a comparative analysis. Rev Esc Enferm USP. 2016;50(1):50-8. doi: 10.1590/S0080-623420160000100007

9. Oliveira ZM, Vargens OMC, Santos RS. Cuidado de enfermagem no climatério: perspectiva desmedicalizadora na atenção primária de saúde. Rev Enferm UFPE On Line [Internet]. 2017 [cited 2020 Jun 10];11(Suppl 2):1032-43. Available from: https://periodicos.ufpe.br/revistas/revistaenfermagem/article/view/13474

10. Treviso P, Peres SC, Silva AD, Santos AA. Competências do enfermeiro na gestão do cuidado. RAS Rev Adm Saúde. 2017;17(69). doi: 10.23973/ras.69.59

11. Sousa SM, Bernardino E, Crozeta K, Peres AM, Lacerda MR. Cuidado integral: desafio na atuação do enfermeiro. Rev Bras Enferm. 2017;70(3):504-10. doi: 10.1590/0034-7167-2016-0380

12. Minayo MCS. O desafio do conhecimento. São Paulo: Hucitec; 2014.

13. Campos RTO, Furtado JP. Narrativas: utilização na pesquisa qualitativa em saúde. Rev Saúde Pública. 2008;42(6):1090-6. doi: 10.1590/S0034-89102008005000052

14. Peixoto LN, Araujo MFS, Egydio CA, Ribeiro FE, Fregonesi CEPT, Carmo EM. Perfil e intensidade de sintomas de mulheres no climatério avaliadas em unidades básicas em saúde em Presidente Prudente. Colloq Vitae. 2015;7(1):85-93. doi: 10.5747/cv.2015.v07.n1.v129

15. Cardoso MR, Camargo MJG. Percepções sobre as mudanças nas atividades cotidianas e nos papéis 
ocupacionais de mulheres no climatério. Cad Ter Ocup UFSCar. 2015;23(3):553-69. doi: 10.4322/01044931.ctoAO0574

16. Fonseca EJNC, Figueiredo Neto JA, Rocha TPO, Nogueira IAL, Melo JB, Melo JB, et al. Síndrome metabólica e resistência insulínica pelo Homa-IR no climatério. Int J Cardiovasc Sci. 2018;31(3):201-8. doi: $10.5935 / 2359-4802.20180009$

17. Caluête MEE, Nóbrega AJS, Gouveia RA, Galvão FRO, Vaz LMM. Influência do estado nutricional na percepção da imagem corporal e autoestima de idosas. Rev Bras Geriatr Gerontol. 2015;18(2):319-26. doi: 10.1590/1809-9823.2015.14062

18. Schmidt PJ, Dor RB, Martinez PE, Guerrieri GM, Harsh VL, Thompson K, et al. Effects of estradiol withdrawal on mood in women with past perimenopausal depression: a randomized clinical trial. JAMA Psychiatry 2015;72:(7):714-26. doi:10.1001/jamapsychiatry.2015.0111

19. Pereira MC, Cárdenas MH. Visión filosófica del cuidado humano en la mujer en la etapa del climaterio. Enferm Cuid Humaniz. 2019;8(1):34-45. doi: 10.22235/ech.v8i1.1795

20. Lomônaco C, Tomaz RAF, Ramos MTO. O impacto da menopausa nas relações e nos papéis sociais estabelecidos na família e no trabalho. Reprod Clim. 2015;30(2):58-66. doi: 10.1016/j.recli.2015.08.001

21. Piecha VH, Ebling SBD, Pieszak GM, Silva MM, Silva SO. Percepções de mulheres acerca do climatério. Rev Pesq Cuid Fundam. 2018;10(4):906-12. doi: 10.9789/2175-5361.2018.v10i4.906-912

22. Ferreira ICC, Silva SS, Almeida RS. Menopausa, sinais e sintomas e seus aspectos psicológicos em mulheres sem uso de reposição hormonal. Ens Ciênc [Internet]. 2015 [acesso em 2020 jan 26];19(2):60-4. Disponível em: https://revista.pgsskroton.com/index.php/ensaioeciencia/article/view/3182/0

23. Santos SMP, Gonçalves RL, Azevedo EB, Pinheiro AKD, Barbosa CA, Costa KNF. A vivência da sexualidade por mulheres no climatério. Rev Enferm UFSM. 2014;4(1):113-22. doi: 10.5902/217976928819

24. Curta JC, Weissheimer AM. Percepções e sentimentos sobre as alterações corporais de mulheres climatéricas. Rev Gaúcha Enferm. 2020;41(Spec No):e20190198. doi: https://doi.org/10.1590/19831447.2020.20190198

25. Santos TT, Barilli SLS, Porciuncula MB, Almeida RSC, Specht AM. Avaliação do risco cardiovascular em mulheres docentes no fim do período reprodutivo. Rev Enferm UFSM [Internet]. 2020 [acesso em 2020 jul 30];10(e38):1-16. Disponível em: https://periodicos.ufsm.br/reufsm/article/view/38513/html

Editora Científica Chefe: Cristiane Cardoso de Paula

Editora associada: Tassiane Ferreira Langendorf 
Necessidades de cuidado de mulheres no climatério com hipertensão: possibilidades de... | 20

Fomento / Agradecimento: Universidade Federal de Santa Maria.

\section{Autor correspondente}

Lara Castilhos

E-mail: laracastilhos23@gmail.com

Endereço: Rua Inácio Domingos Velho, 49 - Centro. Santa Rosa do Sul/SC.

CEP: 88.965-000

\section{Contribuições de Autoria}

1 - Lara Castilhos

Concepção ou desenho do estudo/pesquisa, análise e/ou interpretação dos dados, revisão final com participação crítica e intelectual no manuscrito.

\section{2 - Maria Denise Schimith}

Concepção ou desenho do estudo/pesquisa, análise e/ou interpretação dos dados, revisão final com participação crítica e intelectual no manuscrito.

\section{3 - Laís Mara Caetano da Silva}

Análise e/ou interpretação dos dados, revisão final com participação crítica e intelectual no manuscrito.

\section{4 - Lisie Alende Prates}

Análise e/ou interpretação dos dados, revisão final com participação crítica e intelectual no manuscrito.

\section{5 - Nara Marilene Oliveira Girardon-Perlini}

Análise e/ou interpretação dos dados, revisão final com participação crítica e intelectual no manuscrito.

\section{Como citar este Artigo}

Castilhos L, Schimith MD, Silva LMC, Prates LA, Girardon-Perlini NMO. Necessidades de cuidado de mulheres no climatério com hipertensão: possibilidades de trabalho do enfermeiro. Rev. Enferm. UFSM. 2021 [Acesso em: Ano Mês Dia]; vol.11 e15: 1-20. DOI:https://doi.org/10.5902/2179769242948 